\title{
Association of Changes in Metabolic Syndrome Status With the Incidence of Thyroid Nodules: A Prospective Study in Chinese Adults
}

\author{
Qijun Liang*, Shouyi Yu, Shihui Chen, Yan Yang, Shuhua Li, Chenming Hu, \\ Danxuan Huang, Li Kuang and Dongcai Li
}

Health Management Center, Foshan Hospital of Traditional Chinese Medicine, Foshan, China

\section{OPEN ACCESS}

Edited by:

Yuji Nagayama,

Nagasaki University, Japan

Reviewed by:

Marek Ruchala,

Poznan University of Medical

Sciences, Poland

Roberto Vita

University of Messina, Italy

${ }^{*}$ Correspondence:

Qijun Liang

sam03815@hotmail.com

Specialty section

This article was submitted to

Thyroid Endocrinology,

a section of the journal

Frontiers in Endocrinology

Received: 12 May 2020

Accepted: 17 July 2020

Published: 21 August 2020

Citation:

Liang Q, Yu S, Chen S, Yang Y, Li S, Hu C, Huang D, Kuang $L$ and Li D (2020) Association of Changes in Metabolic Syndrome Status With the

Incidence of Thyroid Nodules: A

Prospective Study in Chinese Adults.

Front. Endocrinol. 11:582.

doi: 10.3389/fendo.2020.00582
Although several cross-sectional studies have shown an association of metabolic syndrome (MetS) with nodular thyroid disease, related prospective studies are scarce. This study investigated the association of MetS with thyroid nodule (TN) incidence in Chinese adults, and explored whether the development of or recovery from MetS is associated with changes in the risk of developing TNs. A total of 4,749 Chinese aged 18-65 years were involved in this 6-year prospective study. The association of MetS with TN prevalence was examined. TN-free individuals at baseline $(n=3,133)$ were further examined. TN incidence rates in groups with different MetS statuses (MetS-free, MetS-developed, MetS-recovery and MetS-chronic) were analyzed. Of all participants, 18.21 and $31.65 \%$ had MetS and TNs, respectively. MetS patients had a higher TN prevalence than the non-MetS group (31.08 vs. $19.81 \%$ in males, $p<0.01 ; 59.52$ vs. $39.59 \%$ in females, $p<0.01$ ). Sex, age and MetS were independent risk factors for TNs. At a median follow up of 5.94 years, the MetS-chronic group (4.37/100 person-years) had a higher risk of TNs (adjusted incidence rate ratio [IRR] $=1.288$ [95\% Cl 1.014-1.636]) compared with the MetS-free group (2.72/100 person-years) in the whole cohort. In males, the MetS-chronic group (3.76/100 person-years) had a higher risk of TNs (adjusted IRR $=1.367$ [95\% Cl 1.017-1.835]) compared with the MetS-free group (2.31/100 person-years). In females, the risk of TNs was significantly higher in the MetS-chronic (6.44/100 person-years) and MetS-developed (6.31/100 person-years) groups compared with the MetS-free group (3.23/100 person-years).

Keywords: metabolic syndrome, thyroid nodules, gender, age, prospective study

\section{INTRODUCTION}

In the past 30 years, thyroid nodules (TNs) with an increasing incidence have become a common thyroid disorder detected by ultrasound in $20-67 \%$ of individuals (1). Advances in diagnostic tools, including high-resolution ultrasonography and computed tomography, may partly explain this increasing trend (2). However, changes in the intrinsic characteristics of the general population are likely to be also involved (3).

Similarly, the incidence of metabolic syndrome (MetS), which is characterized by a cluster of metabolic risk factors (e.g., central obesity, hypertension, hyperglycemia, and dyslipidemia), has been rising exponentially in the last decades due to economic 
development and changes in human diets and lifestyles (4). Since the insulin/insulin-like growth factor axis simulates the proliferation of thyroid follicular cells $(5,6)$, it was hypothesized that compensatory hyperinsulinemia following insulin resistance in previous MetS might be responsible for the rising trend of TNs $(7,8)$. In addition, increased incidence in TNs could be due to growing exposure to certain toxicants that may act as thyroid disruptors (9), and age may be another risk factor for TN development $(10,11)$.

Although several reports have demonstrated an association of MetS with TNs, they were mainly cross-sectional studies designed to evaluate either the prevalence of MetS in individuals with TNs vs. controls (12-15), or, conversely, the prevalence of TNs in MetS patients $(16,17)$. Meanwhile, related prospective studies are scarce.

Therefore, this 6-year prospective study aimed to investigate the association of MetS with the incidence of TNs in Chinese adults. Since the MetS status is known to change dynamically (18-20), we also explored whether the development of or recovery from MetS is associated with an altered risk for TNs.

\section{METHODS}

\section{Study Population}

This population-based prospective cohort study was initiated in January 1st, 2013. Chinese adults aged 18-65 years undergoing a routine medical health check-up at the Health Management Center of Foshan Hospital of Traditional Chinese Medicine were enrolled. Exclusion criteria were: (a) a history of other thyroid diseases, including hyperthyroidism, hypothyroidism, subacute thyroiditis, and Hashimoto thyroiditis; (b) a history of thyroid therapy, including medicines, operation, or radiotherapy for head and neck disease; (c) a history of other endocrine diseases, glucocorticoid treatment, or hormone replacement therapy; (d) chronic diseases (cardiac failure, hepatic, or renal dysfunction), or significant mental or neurological disorders; (e) a history of cancer; (f) pregnancy or lactating in women; (g) exposure to iodinated contrast material in the previous 6 months; (h) a history of taking amiodarone, smoking over 3 months, or drinking in the last 6 months (alcohol intake $>25 \mathrm{~g} /$ day for men and $>15 \mathrm{~g} /$ day for women). The study protocol was approved by the Committee on Human Research at Foshan Hospital of Traditional Chinese Medicine. Written informed consent was obtained from each participant.

\section{Definition and Diagnostic Criteria}

MetS was defined according to the updated National Cholesterol Education Program Panel III criteria for Asian-Americans (21). Thyroid nodules were detected as previously described, with diameters equal to or exceeding $2 \mathrm{~mm}$ (22). Non-alcoholic fatty liver disease (NAFLD) was defined according to the "Diagnostic Criteria of Non-alcoholic Fatty Liver Disease by the Chinese Society of Hepatology" after exclusion of viral or autoimmune liver disease and excessive alcohol consumption (23), based on hepatic ultrasonography (24).

\section{Study Groups}

In the prospective part of this study, the participants were divided into 4 groups according to MetS statuses at the beginning of and after a 6-year follow up: MetS-free (individuals consistently without MetS during follow up), MetS-developed (individuals with newly developed MetS), MetS-recovery (individuals recovering from preexisting MetS), and MetS-chronic (individuals with MetS throughout the study).

\section{Data Collection}

Data on demography, health status, and lifestyle were collected using a standard questionnaire. Body weight and height were measured with the participant dressed in light clothing without shoes. Body mass index was determined as weight divided by height squared $\left(\mathrm{kg} / \mathrm{m}^{2}\right)$. Waist circumference (WC) was measured at the midpoint between the lower edge of the costal arch and the top of the iliac crest in the standing position. After 10 min rest, blood pressure was measured thrice at the right arm, and averaged.

\section{Laboratory Measurements}

Blood samples were collected after overnight fasting at baseline and at the end of the follow-up. Clinical biomarkers, including fasting plasma glucose (FPG), total cholesterol (TC), triglycerides (TG), low density lipoprotein cholesterol (LDL-C), and high density lipoprotein cholesterol (HDL-C) were measured on an automatic analyzer (Hitachi, Tokyo, Japan). Specifically, FPG was measured by the hexokinase/G-6-PDH method, and TG and TC by the peroxidase-chromogen method using kinase-glycerol-3-phosphate and cholesterol esterase-cholesterol oxidase, respectively. LDL-C and HDL-C were measured by the direct one-step method.

\section{Thyroid Ultrasonography}

In the supine position with the hyperextended neck on a pillow, ultrasound examination of TNs was performed, assessing TN number and location, by senior experts on a Bmode high-resolution tomographic ultrasound system (Esaote, Genova, Italy).

\section{Follow Up}

The participants were followed up once a year, with text messages reminding them to return to the hospital for medical examination and providing lifestyle guidance. Individuals with abnormal metabolism were also treated during the follow up visits. The follow-up period was 6 years.

\section{Statistical Analysis}

Continuous variables are mean \pm standard deviation (SD), and categorical variables were presented as number (percentage). T-test, ANOVA and Pearson's chi-square test were performed to analyze differences in means and proportions between or between groups as appropriate. Multivariate binary logistic regression analysis was performed to assess risk factors for TN prevalence at baseline. Poisson regression was applied to calculate incidence rate ratios (IRRs) and 95\% confidence intervals (CIs). All statistical analyses were carried out with SPSS 25 (SPSS, USA). Two-sided $P<0.05$ was considered statistically significant. 
TABLE 1 | Clinical characteristics of subjects according to the MetS status stratified by gender.

\begin{tabular}{|c|c|c|c|c|c|c|c|c|c|}
\hline \multirow[t]{2}{*}{ Parameters } & \multicolumn{2}{|c|}{ Total subjects $(n=4749)$} & \multirow[t]{2}{*}{$P$} & \multicolumn{2}{|c|}{ Males $(n=2525)$} & \multirow[t]{2}{*}{$P$} & \multicolumn{2}{|c|}{ Females $(n=2224)$} & \multirow[t]{2}{*}{$P$} \\
\hline & MetS(-) & MetS(+) & & MetS(-) & MetS(+) & & MetS(-) & MetS(+) & \\
\hline$n$ & 3,884 & 865 & & 1,949 & 576 & & 1,935 & 289 & \\
\hline Age (years) & $36.61 \pm 9.98$ & $41.72 \pm 9.44$ & $<0.001$ & $37.02 \pm 9.90$ & $40.02 \pm 8.97$ & $<0.001$ & $36.20 \pm 10.04$ & $45.11 \pm 9.47$ & $<0.001$ \\
\hline Height (cm) & $163.37 \pm 8.14$ & $165.45 \pm 8.46$ & 0.001 & $168.94 \pm 6.48$ & $169.65 \pm 6.22$ & 0.02 & $157.76 \pm 5.30$ & $157.09 \pm 5.67$ & 0.048 \\
\hline Weight (kg) & $60.54 \pm 10.74$ & $73.30 \pm 11.18$ & $<0.001$ & $67.00 \pm 9.62$ & $78.00 \pm 9.40$ & $<0.001$ & $54.02 \pm 7.33$ & $63.93 \pm 8.16$ & $<0.001$ \\
\hline BMI (kg/cm2) & $22.59 \pm 2.99$ & $26.67 \pm 2.70$ & $<0.001$ & $23.46 \pm 2.96$ & $27.07 \pm 2.54$ & $<0.001$ & $21.71 \pm 2.76$ & $25.89 \pm 2.84$ & $<0.001$ \\
\hline WC (cm) & $81.05 \pm 6.69$ & $89.38 \pm 6.41$ & $<0.001$ & $85.17 \pm 5.85$ & $92.20 \pm 5.06$ & $<0.001$ & $76.90 \pm 4.60$ & $83.77 \pm 4.96$ & $<0.001$ \\
\hline SBP (mmHg) & $117.79 \pm 12.69$ & $134.13 \pm 15.00$ & $<0.001$ & $121.30 \pm 11.98$ & $133.93 \pm 13.37$ & $<0.001$ & $114.26 \pm 12.40$ & $134.53 \pm 17.82$ & $<0.001$ \\
\hline DBP (mmHg) & $70.79 \pm 8.57$ & $81.22 \pm 10.28$ & $<0.001$ & $72.83 \pm 8.55$ & $82.06 \pm 9.95$ & $<0.001$ & $68.74 \pm 8.08$ & $79.55 \pm 10.73$ & $<0.001$ \\
\hline FPG (mmol/L) & $5.31 \pm 0.63$ & $6.15 \pm 1.68$ & $<0.001$ & $5.39 \pm 0.74$ & $6.20 \pm 1.74$ & $<0.001$ & $5.23 \pm 0.49$ & $6.05 \pm 1.55$ & 0.508 \\
\hline TG (mmol/L) & $1.16 \pm 0.90$ & $2.70 \pm 2.71$ & $<0.001$ & $1.41 \pm 1.09$ & $2.96 \pm 3.17$ & $<0.001$ & $0.90 \pm 0.55$ & $2.18 \pm 1.26$ & $<0.001$ \\
\hline TCH (mmol/L) & $4.90 \pm 0.93$ & $5.38 \pm 1.09$ & $<0.001$ & $4.97 \pm 0.91$ & $5.43 \pm 1.14$ & $<0.001$ & $4.83 \pm 0.94$ & $5.29 \pm 0.99$ & 0.01 \\
\hline LDL-C (mmol/L) & $2.84 \pm 0.80$ & $3.17 \pm 0.88$ & $<0.001$ & $2.98 \pm 0.78$ & $3.18 \pm 0.89$ & $<0.001$ & $2.71 \pm 0.80$ & $3.15 \pm 0.86$ & 0.002 \\
\hline HDL-C (mmol/L) & $1.47 \pm 0.24$ & $1.26 \pm 0.20$ & $<0.001$ & $1.38 \pm 0.21$ & $1.24 \pm 0.19$ & $<0.001$ & $1.55 \pm 0.24$ & $1.29 \pm 0.20$ & $<0.001$ \\
\hline UA ( $\mu \mathrm{mol} / \mathrm{L})$ & $346.69 \pm 93.05$ & $411.96 \pm 100.42$ & $<0.001$ & $401.68 \pm 84.11$ & $444.00 \pm 97.03$ & $<0.001$ & $291.30 \pm 64.35$ & $348.10 \pm 72.85$ & $<0.001$ \\
\hline NAFLD, $n(\%)$ & $531(13.67)$ & $520(60.12)$ & $<0.001$ & 409 (20.99) & 387 (67.19) & $<0.001$ & $122(6.30)$ & $133(46.02)$ & $<0.001$ \\
\hline TNs, $n(\%)$ & $1,152(29.66)$ & 351 (40.58) & $<0.001$ & 386 (19.81) & 179 (31.08) & $<0.001$ & 766 (39.59) & 172 (59.52) & $<0.001$ \\
\hline
\end{tabular}

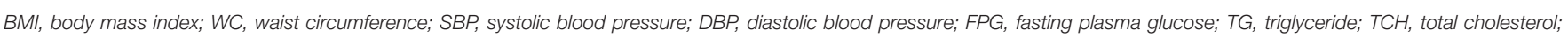

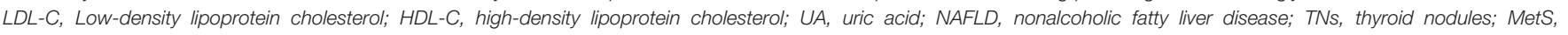
metabolic syndrome.

\section{RESULTS}

\section{General Characteristics of all Subjects and Risk Factors for TNs}

A total of 4,749 subjects were included at baseline. There were 2,525 males and 2,224 females, aged $37.54 \pm 10.07$ years. These participants were stratified by gender and MetS status (Table 1) and further analyzed. In all subjects, the prevalence of MetS was $18.21 \%$. MetS prevalence was significantly higher in males compared with females ( 22.81 vs. $12.99 \%, p<0.01)$.

Based on thyroid ultrasonography, the prevalence of TNs was $31.65 \%$ in the whole cohort, and significantly higher in females than in males $(42.18$ vs. $22.38 \%, p<0.01)$. In addition, the prevalence of TNs was significantly increased in the MetS group compared with MetS-free individuals ( 40.58 vs. $29.66 \%$ in all subjects, $p<0.01 ; 31.08$ vs. $19.81 \%$ in males, $p<0.01 ; 59.52$ vs. $39.59 \%$ in females, $p<0.01$ ). Multivariate logistic regression analysis revealed that sex, age and MetS were independent risk factors for TNs in the whole cohort (Table 2). After stratified analysis by gender, age, and MetS were still independently associated with TN prevalence in both males and females.

\section{Characteristics of the Followed Up Participants}

After thyroid ultrasound, the TN-free subjects were enrolled in a follow up prospective study that ended on December $31^{\text {st }}, 2019$. There were 3,246 participants without TNs at baseline. During the study process, 13 women became pregnant, six individuals had Graves' disease, 27 participants had Hashimoto thyroiditis, one individual had subacute thyroiditis, and 66 subjects quit for personal reasons. Finally, 3,133 participants (1,900 males and
TABLE 2 | Multivariate analysis of potential risk factors for TNs in the whole study cohort.

\begin{tabular}{lcc}
\hline & OR $(\mathbf{9 5} \% \mathbf{~ C l )}$ & $\boldsymbol{P}$ \\
\hline TOTAL SUBJECTS & & $<0.001$ \\
Sex & $2.883(2.522-3.294)$ & $<0.001$ \\
Age & $1.056(1.049-1.063)$ & $<0.001$ \\
MetS & $1.550(1.310-1.832)$ & $<0.001$ \\
MALES & & $<0.001$ \\
Age & $1.041(1.031-1.051)$ & \\
MetS & $1.656(1.339-2.048)$ & $<0.001$ \\
FEMALES & & 0.042 \\
Age & $1.070(1.060-1.080)$ & \\
MetS & $1.326(1.010-1.739)$ & \\
\hline
\end{tabular}

MetS, metabolic syndrome; OR, odds ratio; $\mathrm{Cl}$, confidence interval.

1,233 females) were followed up with a median time of 5.94 years (interquartile range of 5.84-6.05 years).

The baseline (2013) and follow up (2019) data of the 3,133 participants according to MetS status are shown in Table 3. In all participants, the MetS-free group included the highest percentage of females and the youngest population, while the MetS-chronic group included the elderly population. In male participants, the MetS-free group consisted of youngest individuals, while the MetS-recovery and MetS-chronic groups included the elderly population. In female participants, the MetS-free group included the youngest population, and the MetS-chronic group consisted of elderly individuals. 
TABLE 3 | Baseline and follow up characteristics of the participants according to the MetS status, stratified by gender.

\begin{tabular}{|c|c|c|c|c|c|c|c|c|c|c|c|c|c|c|c|c|}
\hline \multirow[t]{2}{*}{ Parameters } & \multirow[t]{2}{*}{ Year } & \multicolumn{4}{|c|}{ Total participants $(n=3,133)$} & \multirow[t]{2}{*}{$\boldsymbol{P}$} & \multicolumn{4}{|c|}{ Males $(n=1,900)$} & \multirow[t]{2}{*}{$P$} & \multicolumn{4}{|c|}{ Females $(n=1,233)$} & \multirow[t]{2}{*}{$\boldsymbol{P}$} \\
\hline & & MetS-free & MetS-developed & d MetS-recovery & MetS-chronic & & MetS-free & MetS-developec & MetS-recovery & MetS-chronic & & MetS-free & MetS-developed & MetS-recovery & MetS-chronic & \\
\hline N & & 2,368 & 261 & 194 & 310 & & 1,306 & 205 & 149 & 240 & & 1,062 & 56 & 45 & 70 & \\
\hline Male, $n(\%)$ & & $1,306(55.15)$ & $205(78.54)$ & $149(76.80)$ & $240(77.42)$ & $<0.001$ & & & & & & & & & & \\
\hline Age (years) & 2013 & $34.71 \pm 9.41$ & $38.36 \pm 10.65$ & $39.91 \pm 9.45$ & $40.47 \pm 9.47$ & $<0.001$ & $35.90 \pm 9.66$ & $37.87 \pm 10.71$ & $39.93 \pm 9.47$ & $39.10 \pm 8.87$ & $<0.001$ & $33.24 \pm 8.87$ & $40.13 \pm 10.31$ & $39.85 \pm 9.47$ & $45.17 \pm 10.00$ & $<0.001$ \\
\hline \multirow[t]{2}{*}{ Height $(\mathrm{cm})$} & 2013 & $164.14 \pm 7.94$ & $167.31 \pm 7.85$ & $167.02 \pm 7.80$ & $167.63 \pm 7.98$ & $<0.001$ & $168.99 \pm 6.36$ & $169.72 \pm 6.57$ & $169.61 \pm 6.56$ & $170.45 \pm 6.06$ & 0.007 & $158.17 \pm 5.12$ & $158.47 \pm 5.48$ & $158.44 \pm 4.94$ & $157.97 \pm 5.96$ & 0.938 \\
\hline & 2019 & $164.31 \pm 7.94$ & $167.56 \pm 7.81$ & $167.26 \pm 7.76$ & $167.90 \pm 7.85$ & $<0.001$ & $169.12 \pm 6.40$ & $169.99 \pm 5.50$ & $169.76 \pm 6.67$ & $170.62 \pm 6.10$ & 0.004 & $158.38 \pm 5.16$ & $158.70 \pm 5.44$ & $158.98 \pm 4.83$ & $158.56 \pm 5.72$ & 0.854 \\
\hline \multirow[t]{2}{*}{ Weight (kg) } & 2013 & $60.14 \pm 10.30$ & $71.48 \pm 10.82$ & $72.65 \pm 9.99$ & $76.67 \pm 10.61$ & $<0.001$ & $65.76 \pm 9.06$ & $73.91 \pm 10.12$ & $75.67 \pm 8.66$ & $79.99 \pm 8.97$ & $<0.001$ & $53.23 \pm 6.99$ & $62.58 \pm 8.40$ & $62.64 \pm 7.21$ & $65.31 \pm 7.53$ & $<0.001$ \\
\hline & 2019 & $61.18 \pm 10.35$ & $74.17 \pm 10.46$ & $71.12 \pm 10.44$ & $77.22 \pm 11.20$ & $<0.001$ & $66.78 \pm 9.14$ & $76.45 \pm 9.42$ & $74.13 \pm 8.96$ & $80.78 \pm 9.44$ & $<0.001$ & $54.30 \pm 7.10$ & $65.81 \pm 9.88$ & $61.16 \pm 8.69$ & $65.00 \pm 7.59$ & $<0.001$ \\
\hline \multirow[t]{2}{*}{$\mathrm{BMI}\left(\mathrm{kg} / \mathrm{cm}^{2}\right)$} & 2013 & $22.23 \pm 2.84$ & $25.46 \pm 2.92$ & $25.97 \pm 2.52$ & $27.20 \pm 2.56$ & $<0.001$ & $23.00 \pm 2.76$ & $25.63 \pm 3.02$ & $26.28 \pm 2.35$ & $27.50 \pm 2.31$ & $<0.001$ & $21.28 \pm 2.65$ & $24.84 \pm 2.41$ & $24.96 \pm 2.77$ & $26.21 \pm 3.08$ & $<0.001$ \\
\hline & 2019 & $22.57 \pm 2.82$ & $26.34 \pm 2.66$ & $25.35 \pm 2.71$ & $27.29 \pm 2.68$ & $<0.001$ & $23.33 \pm 2.75$ & $26.42 \pm 2.62$ & $25.70 \pm 2.47$ & $27.71 \pm 2.48$ & $<0.001$ & $21.64 \pm 2.63$ & $26.03 \pm 2.81$ & $24.18 \pm 3.15$ & $25.87 \pm 2.88$ & $<0.001$ \\
\hline \multirow[t]{2}{*}{ WC (cm) } & 2013 & $80.71 \pm 6.45$ & $87.93 \pm 7.29$ & $89.19 \pm 5.81$ & $91.82 \pm 6.31$ & $<0.001$ & $84.27 \pm 5.49$ & $89.32 \pm 7.20$ & $90.97 \pm 4.81$ & $93.56 \pm 5.48$ & $<0.001$ & $76.33 \pm 4.59$ & $82.85 \pm 5.08$ & $83.32 \pm 4.91$ & $85.85 \pm 5.25$ & $<0.001$ \\
\hline & 2019 & $81.28 \pm 6.47$ & $89.65 \pm 6.74$ & $88.26 \pm 5.98$ & $92.17 \pm 6.43$ & $<0.001$ & $84.86 \pm 5.45$ & $91.06 \pm 6.58$ & $90.05 \pm 4.92$ & $93.98 \pm 5.56$ & $<0.001$ & $76.88 \pm 4.65$ & $84.49 \pm 4.40$ & $82.37 \pm 5.41$ & $85.97 \pm 5.28$ & $<0.001$ \\
\hline \multirow[t]{2}{*}{$\mathrm{SBP}(\mathrm{mmHg})$} & 2013 & $116.70 \pm 11.72$ & $123.08 \pm 11.08$ & $130.59 \pm 13.28$ & $134.38 \pm 14.09$ & $<0.001$ & $120.59 \pm 10.92$ & $124.55 \pm 10.92$ & $132.52 \pm 11.33$ & $134.30 \pm 13.52$ & $<0.001$ & $111.92 \pm 10.87$ & $717.71 \pm 10.00$ & $124.18 \pm 16.94$ & $135.09 \pm 16.00$ & $<0.001$ \\
\hline & 2019 & $118.71 \pm 12.87$ & $132.01 \pm 12.34$ & $127.56 \pm 14.15$ & $136.40 \pm 14.81$ & $<0.001$ & $122.60 \pm 12.22$ & $132.59 \pm 11.85$ & $129.62 \pm 13.55$ & $136.72 \pm 13.33$ & $<0.001$ & $113.93 \pm 12.00$ & $129.89 \pm 13.89$ & $120.71 \pm 14.07$ & $135.33 \pm 19.10$ & $<0.001$ \\
\hline \multirow[t]{2}{*}{$\mathrm{DBP}(\mathrm{mmHg})$} & 2013 & $70.21 \pm 8.20$ & $74.96 \pm 7.86$ & $79.79 \pm 9.39$ & $82.02 \pm 10.38$ & $<0.001$ & $72.28 \pm 8.08$ & $75.73 \pm 7.90$ & $80.97 \pm 8.60$ & $82.60 \pm 10.43$ & $<0.001$ & $67.67 \pm 7.63$ & $72.14 \pm 7.08$ & $75.89 \pm 10.88$ & $80.05 \pm 10.05$ & $<0.001$ \\
\hline & 2019 & $69.94 \pm 8.82$ & $77.31 \pm 9.58$ & $76.49 \pm 10.43$ & $81.96 \pm 11.33$ & $<0.001$ & $72.01 \pm 8.84$ & $78.03 \pm 9.77$ & $78.12 \pm 10.26$ & $82.76 \pm 11.21$ & $<0.001$ & $67.38 \pm 8.10$ & $74.68 \pm 8.42$ & $71.09 \pm 9.17$ & $79.23 \pm 11.41$ & $<0.001$ \\
\hline \multirow[t]{2}{*}{$\mathrm{FPG}(\mathrm{mmol} / \mathrm{L})$} & 2013 & $5.32 \pm 0.48$ & $5.65 \pm 1.27$ & $5.97 \pm 1.00$ & $6.24 \pm 1.55$ & $<0.001$ & $5.38 \pm 0.51$ & $5.69 \pm 1.40$ & $6.06 \pm 1.10$ & $6.13 \pm 1.11$ & $<0.001$ & $5.24 \pm 0.43$ & $5.51 \pm 0.55$ & $5.77 \pm 0.45$ & $6.64 \pm 2.49$ & $<0.001$ \\
\hline & 2019 & $4.99 \pm 0.60$ & $5.49 \pm 1.38$ & $5.37 \pm 1.27$ & $6.23 \pm 2.32$ & $<0.001$ & $5.07 \pm 0.65$ & $5.50 \pm 1.50$ & $5.44 \pm 1.43$ & $6.24 \pm 2.45$ & $<0.001$ & $4.90 \pm 0.52$ & $5.42 \pm 0.74$ & $5.15 \pm 0.39$ & $6.22 \pm 1.80$ & $<0.001$ \\
\hline \multirow[t]{2}{*}{$\mathrm{TG}(\mathrm{mmol} / \mathrm{L})$} & 2013 & $1.07 \pm 0.81$ & $1.95 \pm 1.43$ & $2.37 \pm 1.39$ & $2.90 \pm 1.84$ & $<0.001$ & $1.28 \pm 0.95$ & $2.12 \pm 1.52$ & $2.41 \pm 1.48$ & $3.11 \pm 1.92$ & $<0.001$ & $0.81 \pm 0.49$ & $1.34 \pm 0.72$ & $2.27 \pm 1.06$ & $2.19 \pm 1.33$ & $<0.001$ \\
\hline & 2019 & $1.16 \pm 0.71$ & $2.57 \pm 1.83$ & $1.59 \pm 0.81$ & $2.91 \pm 2.31$ & $<0.001$ & $1.34 \pm 0.83$ & $2.70 \pm 1.97$ & $1.65 \pm 0.86$ & $3.16 \pm 2.43$ & $<0.001$ & $0.95 \pm 0.45$ & $2.07 \pm 1.02$ & $1.39 \pm 0.59$ & $2.09 \pm 1.57$ & $<0.001$ \\
\hline \multirow[t]{2}{*}{$\mathrm{TCH}(\mathrm{mmol} / \mathrm{L})$} & 2013 & $4.82 \pm 0.89$ & $4.96 \pm 0.84$ & $5.40 \pm 0.94$ & $5.38 \pm 1.09$ & $<0.001$ & $4.93 \pm 0.92$ & $4.95 \pm 0.84$ & $5.51 \pm 0.94$ & $5.41 \pm 1.07$ & $<0.001$ & $4.68 \pm 0.83$ & $4.98 \pm 0.87$ & $5.04 \pm 0.87$ & $5.30 \pm 1.18$ & $<0.001$ \\
\hline & 2019 & $4.94 \pm 0.88$ & $5.07 \pm 0.89$ & $5.22 \pm 0.96$ & $5.38 \pm 1.09$ & $<0.001$ & $5.06 \pm 0.89$ & $5.01 \pm 0.86$ & $5.27 \pm 0.99$ & $5.40 \pm 1.07$ & $<0.001$ & $4.81 \pm 0.84$ & $5.29 \pm 0.97$ & $5.06 \pm 0.86$ & $5.33 \pm 1.15$ & $<0.001$ \\
\hline \multirow[t]{2}{*}{ LDL-C(mmol/L) } & 2013 & $2.76 \pm 0.76$ & $2.88 \pm 0.76$ & $3.18 \pm 0.84$ & $3.11 \pm 0.92$ & $<0.001$ & $2.93 \pm 0.78$ & $2.90 \pm 0.77$ & $3.28 \pm 0.82$ & $3.11 \pm 0.93$ & $<0.001$ & $2.55 \pm 0.68$ & $2.83 \pm 0.74$ & $2.86 \pm 0.83$ & $3.12 \pm 0.92$ & $<0.001$ \\
\hline & 2019 & $2.9 \pm 0.77$ & $3.05 \pm 0.81$ & $3.22 \pm 0.84$ & $3.18 \pm 0.96$ & $<0.001$ & $3.08 \pm 0.78$ & $3.03 \pm 0.82$ & $3.30 \pm 0.86$ & $3.16 \pm 0.95$ & 0.005 & $2.68 \pm 0.70$ & $3.12 \pm 0.78$ & $2.96 \pm 0.73$ & $3.24 \pm 0.97$ & $<0.001$ \\
\hline \multirow[t]{2}{*}{$\mathrm{HDL}-\mathrm{C}(\mathrm{mmo} / \mathrm{L})$} & 2013 & $1.49 \pm 0.22$ & $1.33 \pm 0.16$ & $1.33 \pm 0.19$ & $1.29 \pm 0.16$ & $<0.001$ & $1.42 \pm 0.20$ & $1.28 \pm 0.13$ & $1.32 \pm 0.17$ & $1.27 \pm 0.14$ & $<0.001$ & $1.58 \pm 0.22$ & $1.50 \pm 0.17$ & $1.36 \pm 0.24$ & $1.36 \pm 0.19$ & $<0.001$ \\
\hline & 2019 & $1.39 \pm 0.31$ & $1.06 \pm 0.22$ & $1.24 \pm 0.23$ & $1.08 \pm 0.22$ & $<0.001$ & $1.28 \pm 0.27$ & $1.00 \pm 0.18$ & $1.21 \pm 0.21$ & $1.05 \pm 0.20$ & $<0.001$ & $1.52 \pm 0.30$ & $1.26 \pm 0.24$ & $1.32 \pm 0.27$ & $1.21 \pm 0.23$ & $<0.001$ \\
\hline \multirow[t]{2}{*}{$\mathrm{UA}(\mu \mathrm{mol} / \mathrm{L})$} & 2013 & $346.11 \pm 90.66$ & $402.21 \pm 100.51$ & $406.25 \pm 103.39$ & $431.67 \pm 98.90$ & $<0.001$ & $396.43 \pm 79.62$ & $423.03 \pm 96.61$ & $428.08 \pm 101.49$ & $457.60 \pm 90.98$ & $<0.001$ & $284.22 \pm 59.90$ & $325.99 \pm 74.90$ & $333.98 \pm 72.69$ & $342.79 \pm 69.12$ & $<0.001$ \\
\hline & 2019 & $362.48 \pm 93.81$ & $433.83 \pm 84.94$ & $402.77 \pm 97.54$ & $447.60 \pm 96.58$ & $<0.001$ & $413.31 \pm 81.86$ & $451.54 \pm 78.13$ & $422.01 \pm 92.40$ & $471.90 \pm 86.07$ & $<0.001$ & $299.97 \pm 65.57$ & $369.01 \pm 77.57$ & $339.09 \pm 87.24$ & $364.27 \pm 83.74$ & $<0.001$ \\
\hline \multirow[t]{2}{*}{ NAFLD, n(\%) } & 2013 & 254 (10.73) & $98(37.55)$ & $106(54.64)$ & $210(67.74)$ & .001 & $219(16.77)$ & $86(41.95)$ & 93 (62.42) & 173 (72.08) & $<0.001$ & $35(3.30)$ & $12(21.43)$ & $13(28.89)$ & $37(52.86)$ & $<0.001$ \\
\hline & 2019 & $375(15.84)$ & $163(62.45)$ & $85(43.81)$ & $240(77.42)$ & $<0.001$ & $321(24.58)$ & $129(62.93)$ & $72(48.32)$ & $199(82.92)$ & $<0.001$ & $54(5.08)$ & $34(60.71)$ & $13(28.89)$ & $41(58.57)$ & $<0.001$ \\
\hline
\end{tabular}

BMI, body mass index; WC, waist circumference; SBP, systolic blood pressure; DBP, diastolic blood pressure; FPG, fasting plasma glucose; TG, triglyceride; TCH, total cholesterol; LDL-C, Low-density lipoprotein cholesterol; HDL-C, high-density lipoprotein cholesterol; UA, uric acid; NAFLD, nonalcoholic fatty liver disease; MetS, metabolic syndrome. 
TABLE 4 | Risk of TNs according to the dynamic MetS status.

\begin{tabular}{|c|c|c|c|c|c|c|c|}
\hline \multirow[t]{2}{*}{ MetS status } & \multirow[t]{2}{*}{ Event, $n$} & \multirow[t]{2}{*}{ Person-years } & \multirow{2}{*}{$\begin{array}{l}\text { Incidence rate per } \\
100 \text { person-years }\end{array}$} & \multicolumn{2}{|c|}{ Unadjusted model } & \multicolumn{2}{|c|}{ Multivariable model* } \\
\hline & & & & IRR (95\% Cl) & $P$ & Adjusted IRR (95\% Cl) & $\boldsymbol{P}$ \\
\hline \multicolumn{8}{|c|}{ TOTAL PARTICIPANTS } \\
\hline MetS-free & 383 & 14,099 & 2.72 & 1 (reference) & & 1 (reference) & \\
\hline MetS-developed & 55 & 1,567 & 3.51 & 1.187 (0.899-1.568) & 0.227 & 1.142 (0.863-1.513) & 0.353 \\
\hline MetS-recovery & 42 & 1,158 & 3.63 & $1.224(0.894-1.677)$ & 0.207 & $1.094(0.797-1.501)$ & 0.579 \\
\hline MetS-chronic & 81 & 1,855 & 4.37 & 1.530 (1.209-1.937) & $<0.001$ & $1.288(1.014-1.636)$ & 0.038 \\
\hline \multicolumn{8}{|l|}{ MALES } \\
\hline MetS-free & 180 & 7,805 & 2.31 & 1 (reference) & & 1 (reference) & \\
\hline MetS-developed & 34 & 1,234 & 2.76 & 1.049 (0.734-1.499) & 0.792 & $0.979(0.685-1.400)$ & 0.908 \\
\hline MetS-recovery & 32 & 889 & 3.6 & 1.407 (0.975-2.030) & 0.068 & $1.228(0.850-1.775)$ & 0.273 \\
\hline MetS-chronic & 54 & 1,436 & 3.76 & 1.518 (1.130-2.037) & 0.006 & $1.367(1.017-1.835)$ & 0.038 \\
\hline \multicolumn{8}{|l|}{ FEMALES } \\
\hline MetS-free & 203 & 6,294 & 3.23 & 1 (reference) & & 1 (reference) & \\
\hline MetS-developed & 21 & 333 & 6.31 & 1.835 (1.174-2.866) & 0.008 & 1.527 (0.975-2.390) & 0.064 \\
\hline MetS-recovery & 10 & 269 & 3.72 & $1.044(0.555-1.963)$ & 0.895 & $0.822(0.436-1.549)$ & 0.543 \\
\hline MetS-chronic & 27 & 419 & 6.44 & 1.899 (1.275-2.828) & 0.002 & 1.139 (0.751-1.726) & 0.540 \\
\hline
\end{tabular}

IRR, incidence rate ratio; MetS, metabolic syndrome; TNs, thyroid nodules.

*Adjusted for age and sex in all participants. Adjusted for age in the males and females. Age was stratified as 18-30, 31-50 and 51-65 years old.

\section{Risk of TNs According to the Dynamic MetS Status}

In all participants, TN incidence was significantly higher in the MetS-chronic group (4.37/100 person-years) compared with the MetS-free group (2.72/100 person-years) throughout follow up. The MetS-free, MetS-developed, and MetS-recovery groups were similar in TN incidence. Similar results were obtained after adjustment for age and sex (Table 4).

In male participants, TN incidence had the same trend. The MetS-chronic group (3.76/100 person-years) showed a higher incidence compared with the MetS-free group (2.31/100 person-years), which was comparable to the MetS-developed and MetS-recovery groups. After adjustment for age, the MetSfree and the MetS-chronic groups still showed a statistically significant difference.

In females, TN incidence rates were significantly higher in the MetS-chronic (6.44/100 person-years) and MetS-developed groups (6.31/100 person-years) compared with the MetS-free (3.23/100 person-years) and MetS-recovery (3.72/100 personyears) groups. After adjustment for age, no significant differences were found among all groups.

\section{Intergroup Comparisons}

In further comparison between study groups with the same initial MetS status (Table 5), the MetS-developed group showed no significant difference in the risk of TNs compared to the MetS-free group. Idem for the MetS-recovery and MetS-chronic groups. These findings indicated that newly developed MetS did not increase the risk of TNs, which was also not reduced by improving metabolic abnormalities.

Comparing study groups with the same MetS status after follow up, the MetS-recovery, and MetS-free groups showed similar risk of TNs (TN risk not increased by MetS), as well as the MetS-developed and MetS-chronic groups (no difference in $\mathrm{TN}$ risk between new and long-term MetS).

\section{DISCUSSION}

In agreement with previous studies $(16,17)$, the cross-sectional part of the present study demonstrated that nodular thyroid disease was more common in MetS patients, and that older age, female gender, and MetS presence were independent factors affecting TN occurrence. In addition, men with chronic MetS have an increased risk of TNs compared with those consistently free from MetS.

The insulin/insulin like growth factor axis, through its mitogenic effect, stimulates the proliferation of thyroid follicular cells. Studies have shown that elevated circulating amounts of insulin promote the formation and growth of TNs (25-27), even in the pediatric population (28). It is likely that the compensatory hyperinsulinemia, which plays a central role in the pathogenesis of MetS (29), is responsible for the rising trend of nodular thyroid disease. The prospective part of the present study found that men with chronic MetS had higher risk of TNs than those consistently without MetS. Besides cardiovascular diseases and type 2 diabetes, an elevated risk of TNs appears to be another complication of chronic MetS status. However, during a median follow up of 5.94 years, no significant increase in the risk of TNs was found for men between newly developed and chronic MetS cases. Recovery from MetS is associated with positive effects on vascular properties (30) and decreases the risk of major adverse cardiovascular events (31). However, we found no significant decrease in the risk of TNs in MetS-recovery men compared to those with chronic MetS. Longer follow up would enable the 
TABLE 5 | TNs risk comparison between study groups.

\begin{tabular}{|c|c|c|c|c|}
\hline Compared subgroups & \multicolumn{2}{|c|}{ Unadjusted model } & \multicolumn{2}{|c|}{ Multivariable model $^{\star}$} \\
\hline MetS-developed vs MetS-free & $1.187(0.899-1.568)$ & 0.227 & $0.977(0.683-1.397)$ & 0.897 \\
\hline MetS-recovery vs MetS-free & $1.224(0.894-1.677)$ & 0.207 & $1.094(0.797-1.501)$ & 0.579 \\
\hline MetS-chronic vs MetS-free & $1.530(1.209-1.937)$ & $<0.001$ & $1.288(1.014-1.636)$ & 0.038 \\
\hline MetS-recovery vs MetS-chronic & $0.840(0.578-1.219)$ & 0.358 & $0.865(0.596-1.257)$ & 0.447 \\
\hline MetS-chronic vs MetS-developed & $1.230(0.873-1.732)$ & 0.236 & $1.124(0.796-1.587)$ & 0.506 \\
\hline \multicolumn{5}{|l|}{ MALES } \\
\hline MetS-developed vs MetS-free & 1.049 (0.734-1.499) & 0.792 & $0.979(0.685-1.400)$ & 0.908 \\
\hline MetS-recovery vs MetS-free & 1.407 (0.975-2.030) & 0.068 & $1.228(0.850-1.775)$ & 0.273 \\
\hline \multicolumn{5}{|l|}{ FEMALES } \\
\hline MetS-developed vs MetS-free & $1.835(1.174-2.866)$ & 0.008 & $1.527(0.975-2.390)$ & 0.064 \\
\hline MetS-recovery vs MetS-free & $1.044(0.555-1.963)$ & 0.895 & $0.822(0.436-1.549)$ & 0.543 \\
\hline MetS-chronic vs MetS-free & $1.899(1.275-2.828)$ & 0.002 & $1.139(0.751-1.726)$ & 0.540 \\
\hline MetS-recovery vs MetS-developed & $0.589(0.278-1.252)$ & 0.169 & $0.565(0.266-1.201)$ & 0.138 \\
\hline MetS-recovery vs MetS-chronic & $0.577(0.279-1.192)$ & 0.137 & $0.712(0.338-1.497)$ & 0.370 \\
\hline MetS-chronic vs MetS-developed & $1.022(0.578-1.807)$ & 0.941 & $0.809(0.447-1.463)$ & 0.483 \\
\hline
\end{tabular}

IRR, incidence rate ratio; MetS, metabolic syndrome; TNs, thyroid nodules.

*Adjusted for age and sex in all participants. Adjusted for age in males and females. Age was stratified as 18-30, 31-50 and 51-65 years old.

evaluation of the long-term effects of altered MetS status on the risk of TNs.

It was reported that estradiol promotes the growth of thyroid cells (32-36). Estradiol is considered a potent growth factor both for benign and malignant thyroid cells (37-39). Men with TNs show reduced levels of testosterone and sex hormone-binding globulin (SHBG) (40). The gender disparity observed in the association of chronic-MetS with the risk of TNs may be due to the different impact of MetS on endogenous sex hormones. Indeed, elevated estradiol (41) and decreased testosterone and SHBG (42-45) amounts have been reported in men with MetS. Meanwhile, the levels of testosterone and SHBG are increased in women with MetS (46-48), which may blunt the effect of hyperinsulinemia.

A previous report (15) and the cross-sectional part of the present study suggested MetS as an independent risk factor for TN occurrence in women. However, in the prospective part of the present study, the MetS-developed and MetS-chronic groups in women showed no higher risk of TNs compared with the MetS-free group after adjustment for age. In case the positive effect of hyperinsulinemia on TN formation is blunted by the fluctuation of sex hormones, a median follow up of 5.94 years might not suffice to clearly observe the effects of MetS on TN formation. Therefore, long-term follow up studies, also including younger subjects and children are required to clarify this issue. It should be noted that similar thyroid nodule and autoimmune thyroid disease (AITD) rates were found in polycystic ovary syndrome (PCOS) cases and controls (49).

To the best of our knowledge, this is the first prospective study investigating the association of dynamic MetS status with the risk of TNs. However, this study had several limitations. First, the study population might be biased, as all subjects were individuals undergoing routine health checkup as determined by themselves. Secondly, we could not include 2-h postprandial plasma glucose, which is one of the MetS diagnostic criteria, because of the limited time for administering the oral glucose tolerance test (OGTT) and other medical examinations in one session of health checkup. Thirdly, the sample size was relatively small, with a relatively short follow up. Larger prospective studies with longer follow up would enable the evaluation of the long-term effects of MetS on TNs and thyroid cancers. Fourthly, insulin levels, insulin resistance, and waist to height ratio (WHtR) were not assessed in this study. Therefore, insulin, and thyroid and sex hormones should be examined in further studies.

\section{CONCLUSION}

The present study showed that nodular thyroid disease is more common in MetS cases, and older age, female gender and MetS were found to be independent risk factors for TN occurrence. In addition, with a median follow up of 5.94 years, men with chronic MetS showed an increased risk of TNs compared with those consistently free from MetS. 


\section{DATA AVAILABILITY STATEMENT}

The original contributions presented in the study are included in the article/supplementary material, further inquiries can be directed to the corresponding authors.

\section{ETHICS STATEMENT}

The studies involving human participants were reviewed and approved by the Committee on Human Research at Foshan Hospital of Traditional Chinese Medicine. The

\section{REFERENCES}

1. Gharib H, Papini E, Paschke R, Duick DS, Valcavi R, Hegedüs L, et al. American association of clinical endocrinologists, associazione medici endocrinologi, and europeanthyroid association medical guidelines for clinical practice for the diagnosis and management of thyroid nodules. Endocr Pract. (2010) 16 (Suppl. 1):1-43. doi: 10.4158/10024.GL

2. Davies L, Welch HG. Increasing incidence of thyroid cancer in the United States, 1973-2002. JAMA. (2006) 295:21647. doi: 10.1001/jama.295.18.2164

3. Enewold L, Zhu K, Ron E, Marrogi AJ, Stojadinovic A, Peoples GE, et al. Rising thyroid cancer incidence in the United States by demographic and tumor characteristics, 1980-2005. Cancer Epidemiol Biomarkers Prev. (2009) 18:784-91. doi: 10.1158/1055-9965.EPI-08-0960

4. Iqbal AZ, Basharat S, Basharat A, Basharat S. Prevalence of the metabolic syndrome and its component abnormalities among school age Pakistani children. J Ayub Med Coll Abbottabad. (2014) 26:194-199.

5. Kimura T, Van Keymeulen A, Golstein J, Fusco A, Dumont JE, Roger PP. Regulation of thyroid cell proliferation by TSH and other factors: a critical evaluation of in vitro models. Endocr Rev. (2001) 22:63156. doi: 10.1210/edrv.22.5.0444

6. Bidey SP, Hill DJ, Eggo MC. Growth factors and goitrogenesis. J Endocrinol. (1999) 160:321-32. doi: 10.1677/joe.0.1600321

7. Arduc A, Isik S, Ozuguz U, Tutuncu YA, Kucukler FK, Ozcan HN, et al. Relationship between thyroid nodules and non-functioning adrenal incidentalomas and their association with insulin resistance. Endocr Res. (2014) 39:99-104. doi: 10.3109/07435800.2013.840653

8. Tsatsoulis A. The role of insulin resistance/hyperinsulinism on the rising trend of thyroid and adrenal nodular disease in the current environment. JClin Med. (2018) 7:37. doi: 10.3390/jcm7030037

9. Benvenga S, Antonelli A, Vita R. Thyroid nodules and thyroid autoimmunity in the context of environmental pollution. Rev Endocr Metab Disord. (2015) 16:319-40. doi: 10.1007/s11154-016-9327-6

10. Guo W, Tan L, Chen W, Fan L, Chen Y, Du C, et al. Relationship between metabolic syndrome and thyroid nodules and thyroid volume in an adult population. Endocrine. (2019) 65:357-64. doi: 10.1007/s12020-019-01901-4

11. Blanc E, Ponce C, Brodschi D, Nepote A, Barreto A, Schnitman M, et al. Association between worse metabolic control and increased thyroid volume and nodular disease in elderly adults with metabolic syndrome. Metab Syndr Relat Disord. (2015) 13:221-6. doi: 10.1089/met.2014.0158

12. Shin J, Kim MH, Yoon KH, Kang MI, Cha BY, Lim DJ. Relationship between metabolic syndrome and thyroid nodules in healthy Koreans. Korean J Intern Med. (2016) 31:98-105. doi: 10.3904/kjim.2016.31.1.98

13. Chen Y, Zhu C, Chen Y, Wang N, Li Q, Han B, et al. The association of thyroid nodules with metabolic status: a cross-sectional SPECT-China study. Int $J$ Endocrinol. (2018) 2018:6853617. doi: 10.1155/2018/6853617

14. Liu J, Wang C, Tang X, Fu S, Jing G, Ma L, et al. Correlation analysis of metabolic syndrome and its components with thyroid nodules. Diabetes Metab Syndr Obes. (2019) 12:1617-23. doi: 10.2147/DMSO.S219019

15. Ding X, Xu Y, Wang Y, Li X, Lu C, Su J, et al. Gender disparity in the relationship between prevalence of thyroid nodules and metabolic syndrome patients/participants provided their written informed consent to participate in this study.

\section{AUTHOR CONTRIBUTIONS}

QL, YY, SL, CH, and DH carried out the studies, participated in collecting data, and drafted the manuscript. QL, SY, and SC performed the statistical analysis and participated in its design. LK and DL helped to draft the manuscript. All authors read and approved the final manuscript.

components: the SHDC-CDPC community-based study. Mediators Inflamm. (2017) 2017:8481049. doi: 10.1155/2017/8481049

16. Kir S, Aydin Y, Coskun H. Relationship between metabolic syndrome and nodular thyroid diseases. Scand J Clin Lab Invest. (2018) 78:610. doi: 10.1080/00365513.2017.1402363

17. Yin J, Wang C, Shao Q, Qu D, Song Z, Shan P, et al. Relationship between the prevalence of thyroid nodules and metabolic syndrome in the iodine-adequate area of Hangzhou, China: a cross-sectional and cohort study. Int J Endocrinol. (2014) 2014:675796. doi: 10.1155/2014/675796

18. Barceló MA, Rodríguez-Poncelas A, Saez M, Coll-de-Tuero G. The dynamic behaviour of metabolic syndrome and its components in an eight-year population-based cohort from the Mediterranean. PLoS ONE. (2017) 12:e0176665. doi: 10.1371/journal.pone.0176665

19. Fan YC, Chou CC, You SL, Sun CA, Chen CJ, Bai CH. Impact of worsened metabolic syndrome on the risk of dementia: a nationwide cohort study. J Am Heart Assoc. (2017) 6:e004749. doi: 10.1161/JAHA.116.004749

20. Levine GN, Lange RA, Bairey-Merz CN, Davidson RJ, Jamerson K, Mehta PK, et al. Meditation and cardiovascular risk reduction: a scientific statement from the american heart association. J Am Heart Assoc. (2017) 6:e002218. doi: 10.1161/JAHA.117.002218

21. Grundy SM, Cleeman JI, Daniels SR, Donato KA, Eckel RH, Franklin BA, et al. Diagnosis and management of the metabolic syndrome: an American heart association/national heart, lung, and blood institute scientific statement. Circulation. (2005) 112:2735-52. doi: 10.1161/CIRCULATIONAHA.105.169404

22. Cooper DS, Doherty GM, Haugen BR, Kloos RT, Lee SL, Mandel SJ, et al. Revised American thyroid association management guidelines for patients with thyroid nodules and differentiated thyroid cancer. Thyroid. (2009) 19:1167-214. doi: 10.1089/thy.2009.0110

23. Jian-gao F. Guidelines for management of nonalcoholic fatty liver disease: an updated and revised edition. Zhonghua Gan Zang Bing Za Zhi. (2010) 18:163-66.

24. Chartampilas E. Imaging of nonalcoholic fatty liver disease and its clinical utility. Hormones. (2018) 17:69-81. doi: 10.1007/s42000-018-0012-x

25. Rezzonico J, Rezzonico M, Pusiol E, Pitoia F, Niepomniszcze H. Introducing the thyroid gland as another victim of the insulin resistance syndrome. Thyroid. (2008) 18:461-4. doi: 10.1089/thy.2007.0223

26. Sousa PA, Vaisman M, Carneiro JR, Guimarães L, Freitas H, Pinheiro MF, et al. Prevalence of goiter and thyroid nodular disease in patients with class III obesity. Arq Bras Endocrinol Metabol. (2013) 57:1205. doi: 10.1590/S0004-27302013000200004

27. Anil C, Akkurt A, Ayturk S, Kut A, Gursoy A. Impaired glucose metabolism is a risk factor for increased thyroid volume and nodule prevalence in a mild-to-moderate iodine deficient area. Metabolism. (2013) 62:9705. doi: 10.1016/j.metabol.2013.01.009

28. Javier C, Ricardo P, Hugo C. To assess the relationship between hyperinsulinism and thyroid nodules in children with overweight and obesity. Endocrinol Metab Synd. (2015) 4:185-9. doi: 10.4172/2161-1017.1000185

29. Expert Panel on Detection, Evaluation, and Treatment of High Blood Cholesterol in Adults. Executive summary of the third report of the national cholesterol education program (NCEP) expert panel on detection, evaluation, 
and treatment of high blood cholesterol in adults (adult treatment panel III). JAMA. (2001) 285:2486-97. doi: 10.1001/jama.285.19.2486

30. Koskinen J, Magnussen CG, Taittonen L, Räsänen L, Mikkilä V, Laitinen $\mathrm{T}$, et al. Arterial structure and function after recovery from the metabolic syndrome: the cardiovascular risk in young finns study. Circulation. (2010) 121:392-400. doi: 10.1161/CIRCULATIONAHA.109.894584

31. Park S, Lee S, Kim Y, Lee Y, Kang MW, Han K, et al. Altered risk for cardiovascular events with changes in the metabolic syndrome status: a nationwide population-based study of approximately 10 million persons. Ann Intern Med. (2019) 171:875-84. doi: 10.7326/M19-0563

32. Manole D, Schildknecht B, Gosnell B, Adams E, Derwahl M. Estrogen promotes growth of human thyroid tumor cells by different molecular mechanisms. J Clin Endocrinol Metab. (2001) 86:1072-7. doi: $10.1210 /$ jc.86.3.1072

33. Zeng Q, Chen GG, Vlantis AC, van Hasselt CA. Oestrogen mediates the growth of human thyroid carcinoma cells via an oestrogen receptor-ERK pathway. Cell Prolif. (2007) 40:921-35. doi: 10.1111/j.1365-2184.2007.00471.x

34. Kumar A, Klinge CM, Goldstein RE. Estradiol-induced proliferation of papillary and follicular thyroid cancer cells is mediated by estrogen receptors alpha and beta. Int J Oncol. (2010) 36:1067-80. doi: 10.3892/ijo_00000588

35. Rajoria S, Suriano R, Shanmugam A, Wilson YL, Schantz SP, Geliebter J, et al. Metastatic phenotype is regulated by estrogen in thyroid cells. Thyroid. (2010) 20:33-41. doi: 10.1089/thy.2009.0296

36. Saji M, Ringel MD. The PI3K-Akt-mTOR pathway in initiation and progression of thyroid tumors. Mol Cell Endocrinol. (2010) 321:208. doi: 10.1016/j.mce.2009.10.016

37. Costante G, Crocetti U, Schifino E, Ludovico O, Capula C, Nicotera M, et al. Slow growth of benign thyroid nodules after menopause: no need for longterm thyroxine suppressive therapy in post-menopausal women. J Endocrinol Invest. (2004) 27:31-6. doi: 10.1007/BF03350907

38. Kim MH, Park YR, Lim DJ, Yoon KH, Kang MI, Cha BY, et al. The relationship between thyroid nodules and uterine fibroids. Endocr J. (2010) 57:615-21. doi: 10.1507/endocrj.K10E-024

39. Derwahl M, Nicula D. Estrogen and its role in thyroid cancer. Endocr Relat Cancer. (2014) 21:T273-83. doi: 10.1530/ERC-14-0053

40. Chen Y, Chen Y, Wang N, Chen C, Nie X, Li Q, et al. Are thyroid nodules associated with sex-related hormones? A cross-sectional SPECT-China study. BMJ Open. (2017) 7:e015812. doi: 10.1136/bmjopen-2016-015812

41. Maggio M, Lauretani F, Ceda GP, Bandinelli S, Basaria S, Paolisso G, et al. Estradiol and metabolic syndrome in older italian men: the InCHIANTI study. J Androl. (2010) 31:155-62. doi: 10.2164/jandrol.108.006098
42. Corona G, Monami M, Rastrelli G, Aversa A, Tishova Y, Saad F, et al. Testosterone and metabolic syndrome: a meta-analysis study. J Sex Med. (2011) 8:272-83. doi: 10.1111/j.1743-6109.2010.01991.x

43. Brand JS, Rovers MM, Yeap BB, Schneider HJ, Tuomainen TP, Haring R, et al. Testosterone, sex hormone-binding globulin and the metabolic syndrome in men: an individual participant data meta-analysis of observational studies. PLoS ONE. (2014) 9:e100409. doi: 10.1371/journal.pone.01 00409

44. Cunningham GR. Testosterone and metabolic syndrome. Asian J Androl. (2015) 17:192-6. doi: 10.4103/1008-682X.148068

45. Ebrahimi F, Christ-Crain M. Metabolic syndrome and hypogonadism-two peas in a pod. Swiss Med Wkly. (2016) 146:w14283. doi: 10.4414/smw.2016.14283

46. Korhonen S, Hippeläinen M, Vanhala M, Heinonen S, Niskanen L. The androgenic sex hormone profile is an essential feature of metabolic syndrome in premenopausal women: a controlled community-based study. Fertil Steril. (2003) 79:1327-34. doi: 10.1016/S0015-0282(03)00347-9

47. Weinberg ME, Manson JE, Buring JE, Cook NR, Seely EW, Ridker $\mathrm{PM}$, et al. Low sex hormone-binding globulin is associated with the metabolic syndrome in postmenopausal women. Metabolism. (2006) 55:147380. doi: 10.1016/j.metabol.2006.06.017

48. Fatani SH, Abdelbasit NA, Al-Amodi HS, Mukhtar MM, Babakr AT. Testosterone, obesity, and waist circumference as determinants of metabolic syndrome in Saudi women. Diabetes Metab Syndr Obes. (2018) 11:17581. doi: 10.2147/DMSO.S156021

49. Duran C, Basaran M, Kutlu O, Kucukaydin Z, Bakdik S, Burnik FS, et al. Frequency of nodular goiter and autoimmune thyroid disease in patients with polycystic ovary syndrome. Endocrine. (2015) 49:4649. doi: 10.1007/s12020-014-0504-7

Conflict of Interest: The authors declare that the research was conducted in the absence of any commercial or financial relationships that could be construed as a potential conflict of interest.

Copyright (๐ 2020 Liang, Yu, Chen, Yang, Li, Hu, Huang, Kuang and Li. This is an open-access article distributed under the terms of the Creative Commons Attribution License (CC BY). The use, distribution or reproduction in other forums is permitted, provided the original author(s) and the copyright owner(s) are credited and that the original publication in this journal is cited, in accordance with accepted academic practice. No use, distribution or reproduction is permitted which does not comply with these terms. 